\title{
Application of Algorithmic Manifold to Exponential Time
}

Takuya Yabu(takuya.yabu@live.jp)

\section{Introduction}

In the previous paper [1], I defined algorithmic manifolds simulating polynomial-time algorithms, and I showed topological properties for P problem and NP problem [2] and that NP problem can be transformed into deterministic Turing machine problem.

In this paper, I define algorithmic manifolds simulating exponential-time algorithms, and I show topological properties for EXPTIME problem and NEXPTIME problem. I also discuss the relationship between NEXPTIME and deterministic Turing machines.

2. Algorithmic manifold of exponential time

Think about the Riemannian manifold defined by the following metric.

$$
d s^{2}=d n^{2}+O\left(2^{n^{k(l)}}\right) d l^{2}
$$

Here, $n$ is the data capacity to be input to the Turing machine to be processed, and $l$ is the length of the algorithm of the Turing machine to be processed, and $O\left(2^{n^{k(l)}}\right)$ is the time complexity of the algorithm. The Riemannian manifold defined by the above metric is an algorithmic manifold representing an algorithm of exponential time.

From equation( 1 ), the Gauss curvature $K$ is given by the following equation.

$$
K=0
$$

That is, the algorithmic manifold of exponential time is a flat plane. According to the same idea as in the previous paper [1], a connected sum for each branch of a flat plane is homeomorphic to a flat plane, so NEXPTIME and EXPTIME can be said to be homeomorphic. That is

$$
\text { EXPTIME }=\text { NEXPTIME }
$$

Also, arbitrary two points on the algorithm manifold represented by the equation ( 1) are connected by a straight line. Therefore, as for EXPTIME and NEXPTIME as well, it is possible to convert into the problem of deterministic Turing machines, since straight lines can be determined by expressing the start point and end point of the algorithm on the algorithmic manifold expressed by equation (1). Also from this point, equation ( 3 ) holds.

3. Conclusion 
I defined the Riemannian manifold with the metric of equation (1) as an algorithmic manifold expressing the algorithm of exponential time, described the properties derived therefrom, and clarified that EXPTIME = NEXPTIME topologically. I also clarified that the problem of NEXPTIME can be described by deterministic Turing machine.

\section{References}

[1] T. Yabu, "Algorithmic Manifold and Application to P versus NP Problem," IEICE Transactions on Information and Systems, in Posting, https://osf.io/43jcw/, 2016.

[2] S. Cook, “ The P versus NP Problem, http://www.claymath.org/sites/default/files/pvsnp.pdf, 2000. 